\title{
Research on Invisible Grid Management of Information Architecture of UI Design
}

\author{
Yan Liu ${ }^{1, \text { a }}$, Fujun $\mathrm{Ma}^{2, \mathrm{~b}}$ and Fanghao Song ${ }^{3, \mathrm{c}}$ \\ ${ }^{1,3}$ Department of Mechanical Engineering, Shandong University, SDU,Jinan, China \\ ${ }^{2}$ Department of Mechanical Engineering, Weifang College, WDU, Weifang, China \\ a liuyan2008@sdu.edu.cn, ${ }^{\mathrm{b}}$ kagaomei@163.com, ${ }^{\mathrm{c}}$ songfanghao@sdu.edu.cn
}

Keywords: UI design, information architecture, geometry, grid management

\begin{abstract}
Information identification and artistic processing method of UI design has been a hot topic in design field. This paper puts forward a method of invisible grid management of information architecture of UI design. Using design geometry principles, case analysis and other methods, it reduces the levels of UI design, explores the text and image processing method on different carrier interface, improves readability and promotes aesthetic, solves the orderly management of information and data of UI design, which provides a useful reference for design workers.
\end{abstract}

\section{Introduction}

With the development of technology, the selectable mobile terminals increased more rapidly by users. The investigation of UI trends has been designed from materialized to the flat; they flocked to various design trends. How to use visual graphical of user interface breaking boundaries, maximize the information conveying more unified, beautiful, that becomes a hot issue in current UI research, which requires to combine the study of special carrier characteristics of UI and layout design rules .

\section{Analyze main questions of UI design}

About UI design. UI, User Interface, UI design refers to the integrated design of human-computer interaction, logical operating, interface aesthetics of software. Good UI design makes the software becoming individuality and taste, but also makes operation more comfortable, simple and unrestrained, fully reflects the localization and characteristics of software. In essence, UI is a kind of interactive tool in direct contact with user. Because of the special nature of information dissemination carrier, the UI design should follow characteristics of information age, in order to achieve the purpose of good interaction.

There are current personal mobile terminals: mobile phone, pad, computer and any other diverse.

The current main form of UI design. With the popularity of the personal intelligence terminals, the user requirements is not the quality of product itself only, but also product performance including APP software and systems. The requirements of UI design have been increased to accommodate the characteristics of various carriers, that should be proceed in several aspects: optimizing information construction, simplified framework, reduced level relationship according to the needs of the target groups, that benefits user information scan; enhancing interactive experience, improving the aesthetics and ideology of UI, which brings users aesthetic feelings and spiritual experiences.

According to statistics, the proportion of mobile terminal user has an upward trend increased to the major groups. The interface of mobile is smaller than computer screen or pad, and with less operating buttons. Therefore, UI of mobile cannot display a lot of information as web interface, otherwise the text will be too small to read, and advertisements might cause visual confusion, that need to simplify the content and format of information. As a few of operable buttons of mobile, UI designers need to simplify the information construction, reducing levers relationship to suit and facilitate mobile phone users. It can effectively solve above problems by format design. 
The main way of intelligent mobile interaction is via touch gesture and movement sensors. For example, the sensing zone of page-flip gesture of e-book situates corners and sides, slipping to left makes page down, to right makes page back, that making users has real feelings of books reading experience, which is favored by the users. Good interaction design makes user has immersive experience, reflecting the delicate and details of software development, beyond the user's expectations, improve user satisfaction.

The beauty appearance of UI has becomes one of important factors determining the success of UI design currently. According to "the seven seconds law", the direct impression will be produced within first seven seconds when people contact with products. Consequently, the beautiful outlook of UI has more attractive and the fundamental determinants of impression are image colors and construction format, which needs design particularly.

Interface characteristics of UI design. Facing a huge market recently, different styles of UI design emerge in endlessly. How to adapt to different interface layouts of carriers is one of the important issues. The layout design of UI is bones. There are similarities and differences between layout rules of graphic design and UI format design, which can be borrowed by each other for design.

The current UI formats can be divided into vertical version, horizontal version and irregular composite. In computers, for example, the vertical version is usually divided into three or two columns because of the limitations of computer screen and user's visual habit. The horizontal format is usually applied to the interface with big-size captions or predominantly loaded with video files. Modern UI design usually merges the two formats into a compound one. The title bar makes use of the horizontal format while the contents the vertical version or the format of random geometrical forms based on grid.

Due to carrier restrictions of PC, for example, as shown in Fig.1 is a complete interface of UI web version. When users open the home page, the first screen has been earliest seen mainly affecting users 'impressions. Good UI should have show pictures with appropriate size and consistent style, meanwhile the essential information should be displayed completely, and the secondary content link can be displayed on the first screen. Dragged down the home page to display the interface shown in Fig.2, LOGO and other important information can be displayed, show pictures can be ignored.

Key is actually a link portal of information construction. The link key is the basic element of UI design reflecting the overall UI style. The most common link UI buttons component of normal simple graphs and text lack the clear visual identity and aesthetic feature. With the popularity of ios7and ios8, simple color block of flat style becomes in mainstream, but with simple text made it less discriminating pale and monotonous. Thus, the using of image instead of text expression should be the main form. As shown of secondary navigation bar in Fig.2, keys use the background color with the image and text, which made them more eye-catching to enhance identification and interesting.

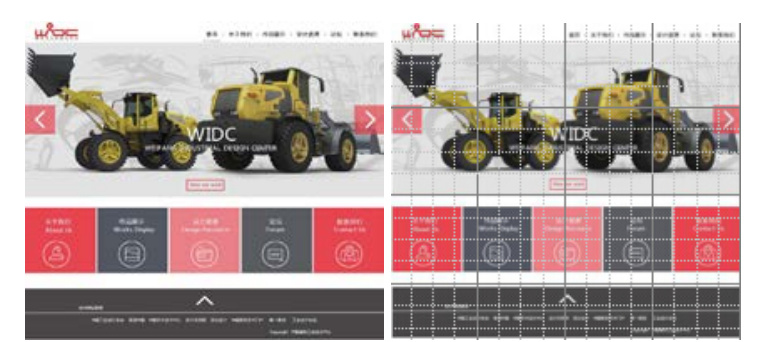

Figure1. The plan form of home page diagram (full version)

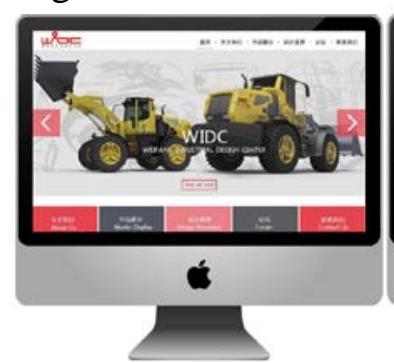

Figure2. The first and last screen of home page interfaces

\section{The application of geometric principles in UI design}

The application of mathematical laws deal with UI design element. (1)The application of the mathematical golden ratio. The golden ratio is a classic rule of variety arts following. In addition to following the law of graphic design, the format design of UI has its own unique transmission, characteristics and unique formatting rules. 
The design elements of UI include images, colors, text and other content, which should be treat as a unity. The format based upon the golden ratio can effectively regulate and optimize the visual effect of UI. As early as 1908, Lalo tested and found people's preferences for length-width ratios of different rectangular items. As shown in Table 1, about 30\% of people prefer these rectangular items conforming to golden section proportion. Thus it is more likely that the proportion resulted from the employment of golden ratio appeal more to people's aesthetic preferences.

Table 1. Lalo Rectangle Preference data[1]

\begin{tabular}{l|lllllllllll}
\hline Width/length & $1: 1$ & $5: 6$ & $4: 5$ & $3: 4$ & $\begin{array}{l}7: \\
10\end{array}$ & $2: 3$ & $5: 8$ & $\begin{array}{l}13: \\
23\end{array}$ & $1: 2$ & $2: 5$ \\
\hline people & $10 \%$ & $1 \%$ & $2 \%$ & $10 \%$ & $5 \%$ & $12 \%$ & $30 \%$ & $8 \%$ & $10 \%$ & $15 \%$ \\
\hline
\end{tabular}

Figure3. Gold section of UI analysis on computer and public number of WeChat example

Figure4. Example of a pad interface

As shown in Fig.3, the computer interface design can be consulted with the golden section derived from golden rectangle, display region accounts for two-thirds of home page. The secondary navigation accounts for one-third position. The logo WIDC and subtitle locate in the middle of two gold rectangles position. According to the golden rectangle layout, it makes the whole UI looks beautiful, coordination and information focused.

The right picture of Fig. 3 is the mobile phone interface of same website of public number of WeChat. Users will subscribe it cause of the favorite after scan. Therefore, publicity and promotional of the public number should be main aim. Compared with the computer interface, information of mobile is more simplified. The main picture is located in the middle position of the golden rectangle, LOGO locates at the top point of the below triangle, and the navigation bar layouts by the top rectangle. Using gold rectangle layout design makes interface more clean and efficient.

There are commonly used of the golden rectangle, triangle constitution ratio and generating a series of circular and square as well as gold spiral. There are many examples of golden ratio utilization in nature, which could be used in UI design.

(2)The application of other proportions. In the natural world, in addition to the golden section, there are many other proportions can be referred to make UI more harmonious and beautiful. For instance, the $\sqrt{2}$ rectangle not only is beautiful and practical, but also can be divided into equal smaller rectangles, which helps to save the cost of printing. As a result, $\sqrt{2}$ rectangle is widely used in graphic design. The ${ }^{\sqrt{3}}$ rectangle can be divided into three equal smaller rectangles. Taking the center of $\sqrt{3}$ rectangle as the center of circle and the $60^{\circ}$ as the distance to rotate three times can obtain regular hexagon, which can be used in the frame-aided of irregular interface format design.

Fig.4 is a pad interface of the same website, using isometric tripartite division of format design puts main information on the first screen without slipping. According to read principle from top to bottom, user should see exhibition information firstly, website business description, and key personnel and specialty, which display information clear and intuitive.

Information architecture of the layout design. The information architecture is based on the importance of the information transmission, massive information and any other elements to arrange format reasonable. As shown in Fig.5, information architecture of web interface systematized and arranged according to importance. Firstly, the information classified into three levels, then designed 
interface framework, navigation, etc. in the light of the priority, which facilitated the rational layout design.

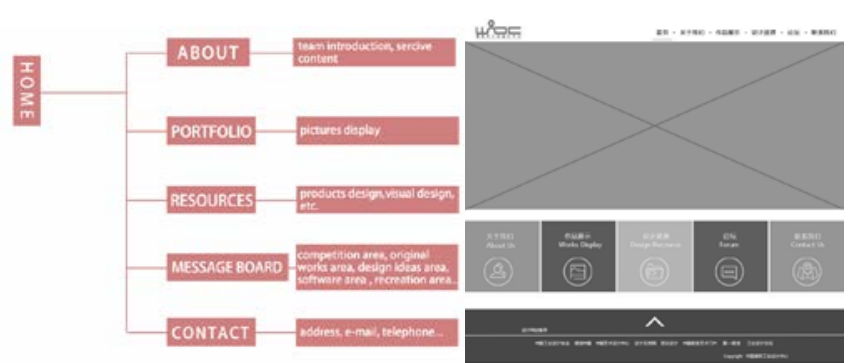

Figure5. The information architecture of web page design

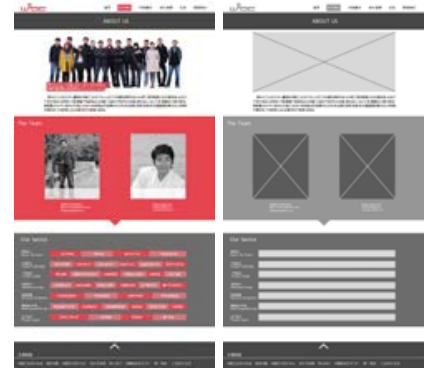

Figure 6. Example of visual rules application

The application of visual rules in UI design. In accordance with the rules of visual design, user brows page from top to bottom usually. Thus, the importance of information is also decremented downward.

This is an important way to combine graphic and text, in order to adapt to the bigger screen of carriers, the utilization of large image becomes a trend. As shown in Fig.6, the top part of interface described a group photo, the character of the image most appropriate to white background. The gray sidebar compared with white background effect harmony, using magenta as the accent color, which suited for logo and ongoing state tips color. The interface using the grid management designed framework, using red, gray, white colors to match each other, which created concise style, variations and harmony of visual effects, achieved the layout design with contrast and similar elements.

\section{Summary}

As the development of technology, the users' visual needs will be diverse in the future. There are problems of information orderly management and artistic technique of aesthetic in UI design need to be solved. Therefore, designers should combine the research results of mathematical geometry, information transmission, visual aesthetic and other multidisciplinary, make design plan for users' visual experience to work out solutions of the humanized and individualized requirements of UI interactions of different carriers.

\section{Acknowledgements}

This work is supported by national social science foundation of China, project number: 13CB114. Key laboratory for high-efficiency and clean mechanical manufacture (Shandong University) Ministry of Education. Shandong University Independent Innovation Fund Project: "The research of GUI design based on mobile Internet applications”, project number: IFW12047.

\section{References}

[1] Kimberly Elam, li Leshan. Trans. "Geometry of Design: Studies in Proportion and Composition,” [M]. 2003.

[2] Ding Yulan. man-machine engineering [M]. Beijing: Beijing Institute of Technology Press, 2007.1.

[3] Liu Yan, Song Fanghao. Design Aesthetics[M]. Hubei: Hubei Fine Arts Publishing House, 2009.

[4] Fujun Ma, Research on WEB Interface Design Based on Visual Experience Theory, Shandong University, (2011)

[5] Fanghao Song,Fujun Ma, Yan Liu. About Five Senses and Six Feelings in Interaction Design[J]. Applied Mechanics and Materials,2012.11. 
[6] Heshan Liu,Fujun Ma. Research on Visual Elements of Web UI Design. IEEE Conference on CIDCD, 2010. 